\title{
Getting value from artificial intelligence in agriculture, over the next 10+ years
}

Submitted to Animal Production Science for the 8th Australasian Dairy Science Symposium, Palmerston North, 21-23 November, New Zealand

\author{
Matthew J. Smith \\ Microsoft \\ London, UK \\ matthew.smith@microsoft.com
}

\begin{abstract}
Every aspect of society and industry seems to be examining the potential benefits from applying artificial intelligence. In this paper I examine the likely benefits to agriculture from applying artificial intelligence and discuss some of the challenges. I end up identifying around 22 different areas, which imply that the highest value capabilities seem to be those where multiple different areas of AI developments are brought together to form new capabilities such as farm robots, digital twins and supply chain traceability.
\end{abstract}

Keywords - Precision, digital twin, traceability, supply chain, robotics

\section{INTRODUCTION}

Artificial intelligence has recently re-entered the public's conscious after impressive demonstrations of what it can now achieve, its incorporation into commonly used digital tools like mobile phones and clear indications by companies that it is a central component to their future products and services . In this article I address how I think agriculture as an industry, and farmers specifically, will benefit over the coming decade from artificial intelligence. I will begin with a more general introduction and some definitions, then I will describe the different areas where I think artificial intelligence will deliver value. In Figure 1 I plot the different innovations covered on a graph indicating my rough personal estimates of relative value and time to value from these different advances and I will refer to this figure as I discuss the different areas. I will also discuss some potentially negative consequences of artificial intelligence as these are also increasingly being considered.

\section{A. Definitions}

Artificial intelligence has existed as a research topic for almost half a century (Buchanan, 2005; Smith \& Shum, 2018) The definition used here is "artificial intelligence is where machines exhibit some degree of intelligence". That makes it reasonably clear that "artificial" is taken to mean a property of a human-made machine. For example, such a machine could be your mobile phone, a satellite navigation system in your tractor, a device in the field or cow-shed - it doesn't matter provided it's giving you or someone some intelligent information or making an intelligent decision on its own.

What is intelligence? Our working definition will be that it is when something is able to perform a new action or changes its state such that it is the optimal response it could perform given external inputs. Note that this doesn't define how that response is arrived at. For example, it may simply select a response, such as an animal feed variety recommendation, from a pre-defined list using a simple algorithm. Alternatively, it could synthesise some new speech through running a much more complex algorithm, e.g. some status update to a farmer in their tractor's satellite navigation system. This indicates that a machine can be an AI even if it's only using very simple computations to give an intelligent response.

\section{B. What's changed recently?}

A set of technological and scientific advances have led to the growth in use of AI in real world applications (Smith \& Shum, 2018)

The first advance has been growth in data about the systems we want to make intelligent decisions about, which itself has arisen because of advances in digital technologies to allow the collection, storage and processing of such data. Historical data allows us to develop AI systems that we can train and test to obtain a measure of confidence that the intelligence they provide is worthwhile.

The second advance has been that the AI algorithms have become a lot more powerful (Smith \& Shum, 2018). This has been underpinned by advances both in the science of $\mathrm{AI}$ and in the computational systems used to build and run them. These new algorithms have learned their intelligent responses through a process of training them to, typically large, training datasets. The result has been the emergence of a new set of algorithms that exhibit advanced cognitive capabilities typically associated with biological organisms such as recognising objects in images, translating speech and deducing the meaning of text. In multiple cases, these algorithms have matched or even exceeded human capabilities at undertaking tasks (e.g. Hassan et al. 2018).

The third advance, which to a large extent underpins the first two, has been almost ubiquitous availability of fast computation; on mobile devices, in sensors, in equipment, personal computers and the cloud. This has meant that data can be acquired easily and information from computation can be delivered in the best possible way to recipients.

The speed of uptake of AI in different industries uptake seems to be linked to the preceding culture and practices of making data-driven decisions. Agriculture, relatively speaking, has been one of the slower adopters. However, that does help us identify some of the AI capabilities that are likely to become popular by allowing us to see what has worked in other industries.

\section{How to think about value from AI}

Ultimately AI systems take data as inputs and do various things in response to it to lead to an output that is useful to someone. They are, therefore, data processing systems. As a result, it is useful when thinking about how value might be 
derived from AI in different circumstances, to be familiar with how value is derived from data analytics.

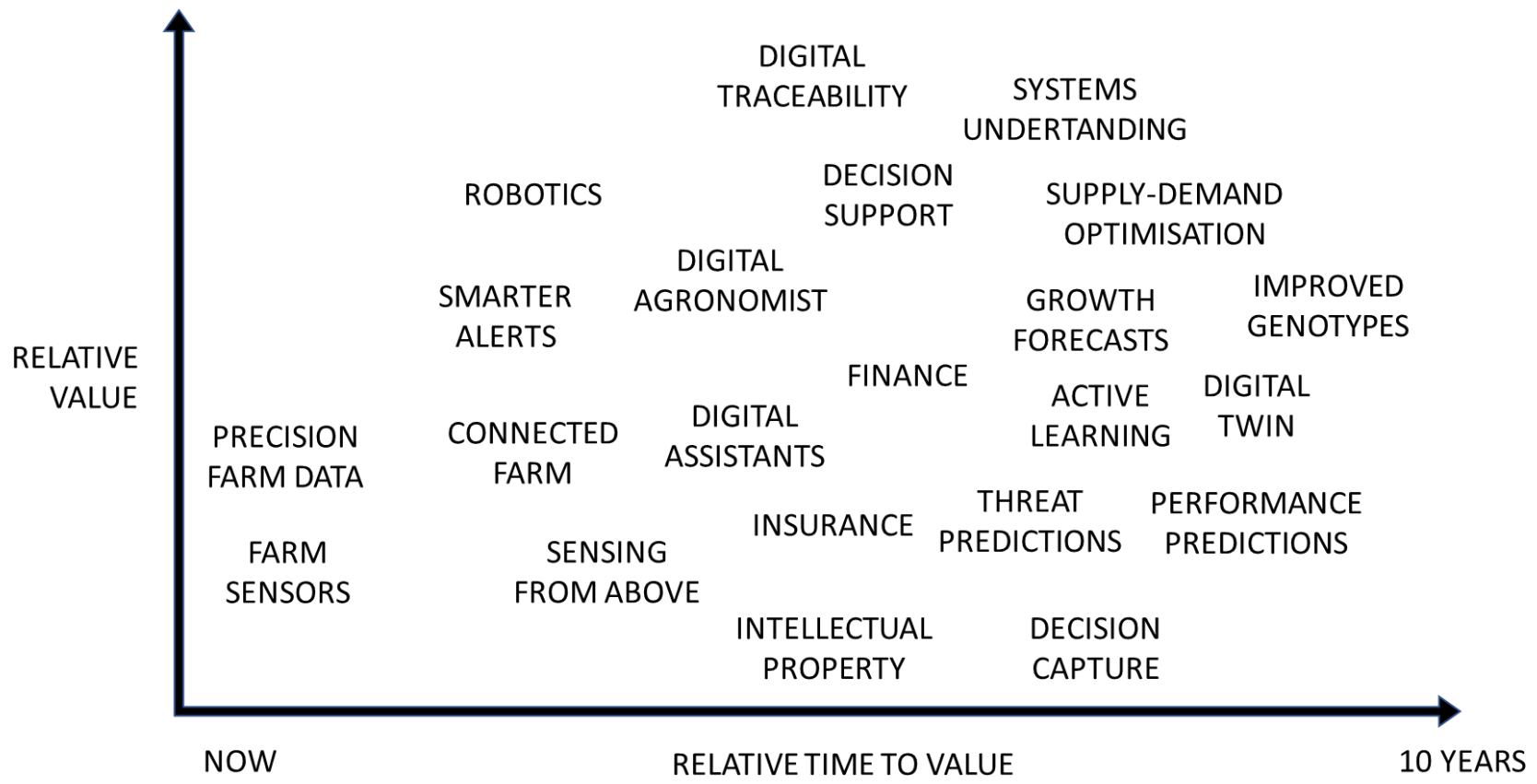

Fig. 1: Artificial intelligence enabled capabilities that will bring value to agriculture over the next decade, with their relative value and time to value approximately estimated by the author.

A common model used to think about deriving value from analytics is the descriptive, diagnostic, predictive, prescriptive spectrum (Banerjee et al. 2013, Fig. 2). The different terms used relate to the role of computation in leading to an action being taken following new inputs.

- In descriptive analytics computation is only used to provide data or information to humans to process and decide. An example in agriculture may be analytics that produce a high spatial resolution field map to indicate variations in forage quality or soil moisture content.

- In diagnostic analytics computation produces relationships between datasets that may imply causality, for example relationships between nutritional components of animal feeds and milk yields or meat quality.

- In predictive analytics computation is used to predict what would happen in space or time. An example would be predicting when crops are likely to be mature enough for harvesting.

- In prescriptive analytics computation is used to make or take recommended actions. An example might be sounding an alarm when an unrecognised person is observed entering a barn.

AI can feature in all these different kinds of analytics and value will be derived for farmers from each. Deriving value from analytics often starts at the descriptive and diagnostic end before enough understanding is generated to know how to deploy predictive or prescriptive analytics.

\section{A. Getting farm data}

Much of the immediate value that will be derived by farmers from AI will be at the descriptive and diagnostic analytics ends of the analytics spectrum. Put simply providing more and better information about the situation on the farm to enable the farmer to look at it, think and decide ("Precision farm data" in Fig. 1). Close or exact analogues are already being realised in much of the precision agriculture applications already deployed on farms such as high-resolution field maps (Vasisht et al, 2017) and animal activity trackers (e.g. Galon, 2010). Modern algorithms are improving on these and beyond them. For example, the significant advances in developing AI algorithms that can visually recognise objects (e.g. cows versus grass, specific weed species) and instances of objects (individual cows, or individual behaviours such as fighting) will improve greatly what can be detected from imagery (He et al. 2016). While the human eye, may often be able to do equally well, it can't always be there: the use of an AI allows this capability to scale to more frequent and more comprehensive monitoring. AI that can detect information from sound or movement or indeed anything that can be sensed by a sensor and turned into a digital signal can also be used to report on farm activities.

AI algorithms can also go beyond processing what humans are constrained to sense, such as chemical signals that may indicate toxins (e.g. Sreelatha et al. 2016). In addition, modern day AI algorithms are being trained to detect information by combining multiple information feeds. For example, Vasisht et al, 2017 showed that aerial imagery could significantly reduce the quantity for soil moisture sensors needed to produce a high-resolution soil moisture 


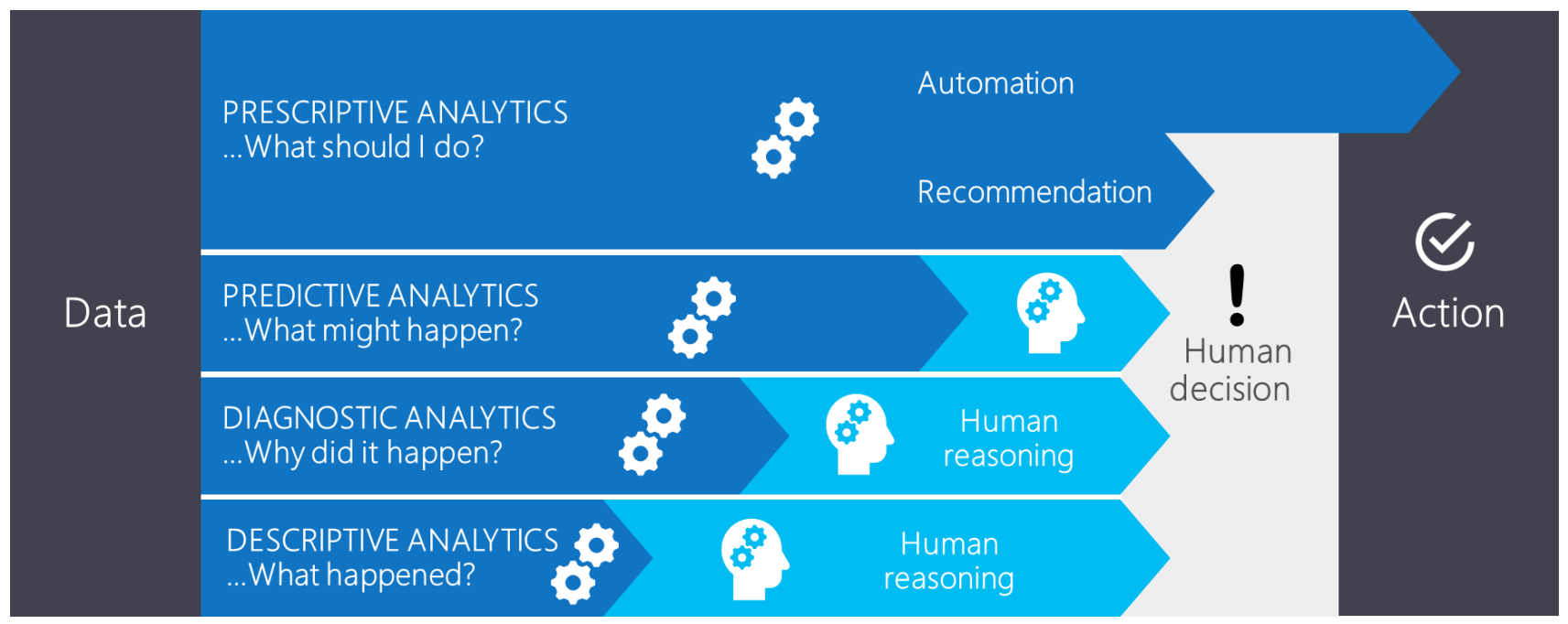

Fig. 2: Transforming data into actionable information through different sorts of computational analytics and requiring different degrees of human information processing.

map when combined with soil moisture meter data coming from AI.

A major application of AI in getting farm data recently has been putting movement sensors and other devices on, and even in, farm animals to enable reporting of their behaviours, health and condition ("Farm sensors", Fig. 1). One of the earliest examples is pedometers to detect estrus in cows (Galon, 2010), which can both reduce manual labour of having to watch cows but also can improve insemination timing so as to maximise the chances of getting a female or male calf.

A common challenge farm data collection is the lack of digital connectivity. Major advances have been made in improving connectivity in remote areas, in improving remote sensing and in enabling AI algorithms to be deployed directly to remote devices (Vasisht et al, 2017). This progress leads me to expect that the problem of a lack of connectivity for AI to be to be largely eliminated within a decade ("Connected farm", Fig. 1). A key advantage of being able to deploy AI algorithms to a device over the internet is that the algorithms can improve their existing capabilities or be programmed with entirely new capabilities.

AI is also being used to improve information extraction from satellite and aerial imagery (Sirosh, 2018). These typically require significant computational costs and domain expertise to turn imagery into meaningful information, such as information about a farmer's fields. Over the coming decade computation and AI will largely automate these steps, making it possible to obtain and record information on any properties that can be detected or inferred from such imagery ("Sensing from above", Fig. 1).

Another application of AI in agriculture will be to help farmers to obtain the information they need in the right way. For example, language translation AI can deliver to farm workers the information they want in the language that works best for them, such as helping in the delivery of agronomic advice to farmers working in areas where people speak different languages. Such capabilities could greatly improve the spread of good agronomic advice which could lead to improved agricultural practices more widely ("Digital agronomists", Fig. 1). Chat-bots, where the interacting agent is simply a machine, could also help farmers to receive the information they need.

AI algorithms to translate and extract knowledge (e.g. Wang et al 2017) can also be used to help with capturing information from farmers and farm workers - such as spoken instructions, requests, observations or even measurements. Such methods are necessary when specific data, such as the observation of the presence of specific pests, needs to be extracted from a continuous stream of unstructured data such as speech. Such capabilities could also help farmers perform tasks in environments where it is only possible to provide spoken instructions, such as when both hands are otherwise being used ("Digital assistants", Fig. 1).

What could be negative consequences of using AI to provide farm data? Leaving the interpretation and the decision making to the farmer/other human reduces negative risks associated with computers making the wrong decisions on their own (although see below for recommender engines). However, in cases where manual labour is used to perform data collection and inspection, then there is clearly a potential disruption from those tasks being completed by computers instead. As has been argued by others, that doesn't always mean those roles need to be removed, just that the human responsibilities change (Smith \& Shum, 2018).

There will continue to be important roles for farm workers in being present in the field because a downside of entirely separating the worker from the full sensory experience of being on location is that it negatively impacts the opportunity to detect new things and make insights.

A challenge that arises with the growth in new information from AI, especially when such systems are data that require human interpretation, is the requirement to train people to interpret the outputs. This can be facilitated by improving the interaction design of the system, but new learning is still going to be required. I especially see this as an opportunity for new trainees coming into agriculture because I think such skills will be increasingly valuable, not just for interpreting new information but also for developing 
better AI systems for the future ("Intellectual property", Fig. $1)$.

\section{B. New and better alerts}

Moving from descriptive to prescriptive analytics: once an ability to record some actionable information has been shown, it is then very typical to attempt to extend that to developing computational capabilities that automate processes or perform further analytics. The most basic of these is issuing alerts when something notifiable is detected ("Smarter alerts", Fig. 1). For example, motion detectors of animals indicating their behaviours tend to get followed by capabilities that provide notifications of key behaviours or patterns. A downside of computational alerts is the risk of over-reliance or over-dependency on them. There will always be the risk of something getting past the computer's senses, just as there is for human senses. As a result, it will always be important to maintain a role for human inspection and testing.

An important aspect of algorithms, including AI, is that their accuracy can drift over time because of the properties of what they are trying to predict itself changing over time. For example an acoustic alerting system in a cow shed listening for animal distress may have worked fine before but because of a new object in the shed changing the acoustics the algorithm no longer reliably detects what it did before. It is therefore important to ensure that reliable systems are put in place to test the accuracy of such alerting systems.

\section{Improved diagnostics}

A downstream consequence of being able to measure and monitor agricultural systems better is that it increases our abilities to investigate and understand the reasons for why various phenomena are occurring. A challenge with biological systems is that may not behave as expected because they are influenced by known or unknown factors in ways we do not understand. Modern large-scale agricultural practices have partially addressed that problem by simplifying the agricultural systems themselves. The collection of more and better data via AI will enable improved analyses of how different factors interact to influence properties of agricultural systems ("Systems understanding", Fig. 1). An implication of improved data capture for diagnostic analysis is that there will need to be people who interpret the outputs and produce actionable insights, which I'm sure will continue to be the case and, indeed, is an example of a potentially positively impacted job role in agriculture caused by recent improvements in AI.

One characteristic of the more advanced AI algorithms is that when there is enough data they can sometimes be trained to successfully predict complex phenomena, accounting for a wide range of interacting factors, in ways what we do not fully understand. This situation understandably causes some nervousness, because it could be argued that if we don't understand why the algorithm is successful we also don't understand its weaknesses and where and why it might fail. A major benefit of $\mathrm{AI}$ in such circumstances however is that it can show that various factors can indeed be used to obtain predictive accuracy where previously it was not known whether it existed. This is a great incentive to investigate why it is that the various factors combine to lead to the observed phenomenon.

\section{Improved predictions}

As data, AI algorithms and biological understanding have improved, so too has the ability to predict the development of produce and farm properties over time and space. A major area of improvement will be predictions about how different varieties of plants and animals will perform in different circumstances ("Performance predictions", Fig. 1). This is the classic consideration of production as a function of genotypic, environmental and management (GxExM) effects, a formula which agricultural research organisations have approximately solved to recommend varieties (Anderson, 2010). With AI, this equation could be solved with more accuracy than ever before, but also delivered to farmers on demand.

Another area of development has been producing real time forecasts of crop or animal status as they develop: factors such as size, development stage and nutritional status ("Growth forecasts", Fig. 1). This requires a GxExM equation to be developed that takes inputs, including uncertain quantities such as weather forecasts, on an ongoing basis, that are themselves updated over time so that the current forecast is continually up to date, much like a weather forecast (Caldararu et al. 2017). Such a capability would enable farmers to optimise their activities. For example, forecasts of likely availability of harvestable plants over the year might lead a farmer to plant more now to make up for a forecasted shortfall.

Producing accurate real time forecasts data-intensive, not just requiring sufficiently accurate local data about the environment and crop or animal management but also up to date data on the crop or animal status. As a result, the effort in making such forecasts is unlikely to always be justifiable. They are certainly valuable in large scale operations that aim to guarantee a predictable pattern of supply of produce to retailers, which certainly covers a lot of agricultural production.

Just as producers will be able to better forecast their production and its properties, AI will also help processors, retailers and wholesalers better forecast their consumption and what is likely to sell. Major efficiency savings could be made by bringing these together to ensure that supply matches demand as best as possible to minimise waste ("Supply-demand optimisation", Fig. 1).

Beyond the individual farm, larger spatial scale predictions of crop and animal development and production are valuable for purchasers of agricultural produce or those impacted by (or banking on!) variations in crop production. Crop pricing, insurance, reinsurance and trade finance have been factoring in production forecasts into their businesses for a long time. However, I think that advances in data availability and AI will enable agricultural insurance far better tailored to individual circumstances and competitive ("Insurance", Fig. 1).

Beyond the actual produce, AI predictions will also increasingly be used to provide indications of the overall health of the farming system and of potential threats that farmers may want to look at more closely or action on. For example, AI inferences of soil water balance and forecasts can help to optimise irrigation and help warn against potential flooding or soil erosion (Belayneh \& Adamowski, 2018; Fotovatikhah et al, 2018; Morgan \& Nearing, 2016). For soil health, predictions of status could help farmers better 
manage the health of their soils and take remedial action in relation to deteriorating soils before problems become acute. Emerging threats are also increasingly predicted such as threats of fungal, insect and plant pests (e.g. Yan et al. 2015).

The improvement predictive information brought about by $\mathrm{AI}$ is transforming predictions from metrics that farmers then translate into threats e.g. wind speed into potential for crop damage, into direct predictions of the threats themselves e.g. predicted extent of drought, levels of oyster contamination, pollination success. As a result I expect the emergence of systems for farmers that don't just provide a few maps of things like weather predictions but instead a whole stack of different predictions of properties of direct relevance to the farmer (Threat predictions", Fig. 1).

This last example naturally suggests a problem that can emerge when there is so much meaningful information that could be presented to a decision maker, that it becomes too much and the decision maker simply chooses to ignore most of it or becomes overwhelmed or annoyed. In such circumstances it is then natural to look for ways to simplify the information and focus the decision maker on the core priority information that they should look at. This lends itself to prescriptive analytics which is covered in the next section.

Another challenge with presenting predictions is ensuring that the decision maker makes the optimal decision. When considering what to do, it is important to consider the uncertainty in the predictions, and efforts continue to improve the quantification and communication of uncertainty in outputs of predictive models. However, it is recognised that people struggle to interpret such uncertainties in a way that leads to the optimal decision (e.g. Retzbach \& Maier, 2015). I think this will have a major influence on what predictions are provided directly to farmers and how they are provided. The tendency will be to pair or even replace predictions with consequences of different possible actions in light of different decisions, which itself requires even more data and AI development.

\section{E. Recommendation systems}

As discussed already, a natural extension of systems that serve data and predictions to farmers is then systems that recommend to the user actions that they could take. This obviously becomes valuable in situations where the farmer is not aware of the different options that are available and the advantages and disadvantages of different choices. A major application of this could be in enabling smallholder farmers to obtain agronomic advice. I think such systems will accelerate improvements in agricultural production in smaller and more remote farms where traditionally there has been more limited access to agronomic advice ("Digital agronomist", Fig. 1). I also think the availability of such systems could help in the funding of aid programs to improve production: a major barrier to aid funding is knowing confidently what help is needed, for what result, when and where ("Finance", Fig. 1)

Another application for recommendation systems, as alluded to in the previous section, is helping decision makers decide what to do when there are multiple objectives that are often competing. Classically, for example, farmers must make decisions in production to maximise profit, quality, farm health, animal health, long term sustainability and other factors that change over different timescales. Increased data on all these factors and the ability to predict their changes and interactions over different timescales opens the promise of managing the farm, or even wider areas, to best meet multiple objectives. This is typically a hard optimization problem that even current computational capabilities can struggle with. AI systems will emerge that will enable decision makers to explore recommended practices and their consequent costs and benefits given the different preferences they give (or want to experiment with). This will start with simple tradeoffs such as between production rates, quality, soil health and costs and extend to longer term farm or landscape management strategies ("Decision support", Fig. $1)$.

A more recent development that will lay the foundations for improved recommendation systems will be systems that capture the action or decision that the farmer did take considering the information that was provided to them. Such data is crucial to improving such systems in future and could be acquired through directly querying the farmer or using automated methods ("Decision capture", Fig. 1). A challenge will be designing such systems so that the farmer feels they receive value from such participation, or at least are not being detrimentally impacted or even exploited.

An emerging area of recommendation systems in general is that of active learning, in which the algorithm recommends the data it needs in order to become more accurate or useful (Settles, 2012). Active learning could enable efficiency gains in the development of better algorithms and could also make the process of interacting with the AI more rewarding for the user. A hypothetical example of active learning would be a system that identifies which moisture measurements should be made where and when in a system of fields to obtain spatial maps and dynamics of water availability. Such a system would be able to minimise (or even simply provide an estimate) the expense of data collection given a desired level of accuracy ("Active learning, Fig. 1).

\section{F. Robotics and automation}

Like in other industries, AI is already having impacts in agriculture through improving robotics and mechanisation (Duckett et al. 2018; "Robotics", Fig. 1). Farm robotics particularly employ prescriptive analytics and the ongoing advances in AI will enable such robots to be useable in an increasingly wide range of circumstances. Driverless farm vehicles, variable application rate planters, precision spraying, precision picking, smart processing and packing systems, and self-managing irrigation systems are just some examples of current industry growth areas.

Robotics is also an area of concern when it comes to negatively impacting farm jobs and I expect that it will force many farm jobs to change. In other cases, robots may be needed because of a lack of available labour. As discussed, I hope that increasingly the laborious repetitive tasks will be carried out by the robots and the human presence will still be required to problem solve and innovate. Another concern with the race to further automate farming is that it could encourage the development of agricultural systems that are more industrial and less sustainable. Present day agricultural robots work best in environments where the terrain is relatively flat, the crops relatively simply structured and the early dominance of such systems without demanding that they work in more complex environments could allow less 
sustainable farming systems to dominate for longer at the expense of more sustainable systems.

\section{G. Supply chain traceability}

A key enabler of improvements in agriculture over the next ten years is going to be the drive towards improvements in supply chain traceability, something increasingly wanted by companies and the public. This is relevant because AI is just the capability needed to unlock a lot of the benefits intended from improving supply chain traceability ("Digital traceability", Fig. 1), such as, for example, recommending adjustments in what is produced (e.g. planted, slaughtered) and to what criteria (e.g. weights of apple) in order to best meet demand and minimise wastage.

There are multiple challenges in achieving supply chain traceability, particularly enabling data between multiple organisations to be linked, while still respecting information access rights and confidentiality. The full analytics spectrum, heavily leveraging AI will be key to overcoming such challenges. For example, with respect to information access and confidentiality, AI will help to assemble supply chain information relevant to a request and potentially highlight and then manage the data access permission requests needed to ensure requestors can use such data.

The development of improved supply chain traceability may just be the right solution for enabling farmers to unlock a lot of the value promised from AI, and from having more data on their farms more generally. Crucially, it enables supply chain organisations to be clearer about the value derived from acquiring and using more data, which will unlock more investment by companies within and even outwith the supply chain to support further improvements. This means that farmers will also be able to get more value from sharing their data: about their farms, produce and practices, even as continuous real-time data feeds (e.g. for animal welfare reasons Berkmans, 2014).

\section{H. Digital Twin}

As time goes on the amount of data about any one entity on a farm increase. An emerging paradigm around which to organise such information is the "digital twin", a digital representation of a specific real-world entity, such as a specific cow, field or even a specific farm. It is relevant here because AI will be key to deriving a lot of the value from digital twins for agricultural systems ("Digital twin", Fig. 1). Digital twins provide an organising system to identify the best response to a specific query. Such digital twins wouldn't just enable access to the historical and near real-time status of entities, but also predictions about those entities. Such predictions could also be under "business as usual" or even considering interventions. For example, a digital twin of a cow could indicate its probability for developing mastitis as a function of various management and treatment decisions.

Digital twins are already being used in farm applications, such as applications that enable farmers to interpret information coming from devices deployed on animals, but further development of the capability is needed. One is to enable the multiple information sets about any one entity to be brought together to simplify information access for the farmer and to enable new insights from combining information from multiple sources. Another is to enable the two-way exchange of information and algorithms between digital twins and the broader population-level data so that insights can be derived from the population level data and then tailored to the individual through the digital twin.

\section{CONCLUSIONS}

I have covered a range of areas in which I believe AI will deliver value in agriculture. In Fig. 1 I have plotted these topics on axes of value and time to value using only my own subjective judgement. This highlights that the highest value capabilities appear to be those in which multiple different areas of AI applications are brought together, such as robotics, digital traceability, and decision support. In going through these different areas, it also became clear that many of the capabilities will build on from or complement each other. As a result, I strongly encourage farmers and agricultural organisations to consider how AI can help them in their business and start at the most accessible entry point. The fact that these capabilities build on each other to deliver increasing value gives me believe that the continued application of AI in agriculture will be one way humanity will address many of the challenges it faces in finding a sustainable future. Clearly also, AI adoption will bring challenges, but it appears each challenge seems to hide a new opportunity for value. Hence, I'd encourage those in agriculture to approach the opportunity of incorporating AI, including the challenges, with enthusiasm.

\section{ACKNOWLEDGEMENTS}

I thank Claudia Roessler and Giri Tharmananthar for comments and advice on the manuscript.

\section{REFERENCES}

Anderson, W.K., 2010. Closing the gap between actual and potential yield of rainfed wheat. The impacts of environment, management and cultivar. Field Crops Research, 116(1-2), pp.14-22.

Banerjee, A., Bandyopadhyay, T. and Acharya, P., 2013. Data analytics: Hyped up aspirations or true potential?. Vikalpa, 38(4), pp.1-12.

Belayneh, A. and Adamowski, J., 2018. Drought Forecasting: Artificial Intelligence Methods. In Exploring Natural Hazards (pp. 207-224). Chapman and Hall/CRC.

Berckmans, D., 2014. Precision livestock farming technologies for welfare management in intensive livestock systems. Scientific and Technical Review of the Office International des Epizooties, 33(1), pp.189-196.

Buchanan, B.G., 2005. A (very) brief history of artificial intelligence. Ai Magazine, 26(4), p.53.

Caldararu, S., Purves, D.W. and Smith, M.J., 2017. The impacts of data constraints on the predictive performance of a general process-based crop model (PeakN-crop v1. 0). Geoscientific Model Development, 10(4), pp.1679-1701.

Fotovatikhah, F., Herrera, M., Shamshirband, S., Chau, K.W., Faizollahzadeh Ardabili, S. and Piran, M.J., 2018. Survey of computational intelligence as basis to big flood management: challenges, research directions and future work. Engineering Applications of Computational Fluid Mechanics, 12(1), pp.411-437. 
Galon, N., 2010. The use of pedometry for estrus detection in dairy cows in Israel. Journal of Reproduction and Development, 56(S), pp.S48-S52.

Hassan, H., Aue, A., Chen, C., Chowdhary, V., Clark, J., Federmann, C., Huang, X., Junczys-Dowmunt, M., Lewis, W., Li, M. and Liu, S., 2018. Achieving human parity on automatic chinese to english news translation. arXiv preprint arXiv:1803.05567.

He, K., Zhang, X., Ren, S. and Sun, J., 2016. Deep residual learning for image recognition. In Proceedings of the IEEE conference on computer vision and pattern recognition (pp. 770-778).

Morgan, R.P.C. and Nearing, M. eds., 2016. Handbook of erosion modelling. John Wiley \& Sons.

Retzbach, A. and Maier, M., 2015. Communicating scientific uncertainty: Media effects on public engagement with science. Communication Research, 42(3), pp.429-456.

Settles, B., 2012. Active learning. Synthesis Lectures on Artificial Intelligence and Machine Learning, 6(1), pp.1-114.

Sirosh, J., 2018, July. Planet-Scale Land Cover Classification with FPGAs. In Proceedings of the 24th ACM SIGKDD International Conference on Knowledge Discovery \& Data Mining (pp. 2877-2877). ACM.
Smith, B \& Shum, H., 2018 The Future Computed: Artificial Intelligence and its Role in Society. Microsoft Corporation, Redmond, Washington, USA

Smith, M., 2018. White paper-Agricultural Robotics: The Future of Robotic Agriculture.

Sreelatha, M., Nasira, G.M. and Thangamani, P., 2016, March. Patten recognition for toxic gases based on electronic nose using artificial neural networks. In Computing for Sustainable Global Development (INDIACom), 2016 3rd International Conference on (pp. 3075-3079). IEEE.

Vasisht, D., Kapetanovic, Z., Won, J., Jin, X., Chandra, R., Sinha, S.N., Kapoor, A., Sudarshan, M. and Stratman, S., 2017, March. FarmBeats: An IoT Platform for Data-Driven Agriculture. In NSDI (pp. 515-529).

Wang, W., Yang, N., Wei, F., Chang, B. and Zhou, M., 2017. Gated self-matching networks for reading comprehension and question answering. In Proceedings of the 55th Annual Meeting of the Association for Computational Linguistics (Volume 1: Long Papers) (Vol. 1, pp. 189-198).

Yan, Y., Feng, C.C., Wan, M.P.H. and Chang, K.T.T., 2015, October. Multiple Regression and Artificial Neural Network for the Prediction of Crop Pest Risks. In International Conference on Information Systems for Crisis Response and Management in Mediterranean Countries (pp. 73-84). Springer, Cham. 